\title{
Chemical Imaging of Live Cancer Cells in the Natural
}

\section{Aqueous Environment}

Marina K. Kuimova, ${ }^{1}$ K. L. Andrew Chan $^{2}$ and Sergei G. Kazarian ${ }^{2 *}$

${ }^{1}$ Department of Chemistry, Imperial College London, South Kensington Campus, SW7

2AZ, United Kingdom

${ }^{2}$ Department of Chemical Engineering, Imperial College London, South Kensington Campus, SW7 2AZ, United Kingdom

Keywords: Living cells, FT-IR Imaging, ATR-FTIR image, cell organelle, label free imaging, cell death. 


\section{ABSTRACT}

Chemical imaging with FTIR spectroscopy allows the visualisation of the distribution of chemical components in cells without the need for labels or added dyes. However, obtaining such images of living cells is difficult because of the strong absorption of water in the mid-infrared region.

We report the use of Attenuated Total Reflectance (ATR) FTIR spectroscopic imaging to study live human cancer cells in an aqueous environment, on a single cell level. Two complementary approaches have been used providing flexibility with field of view and spatial resolution: (i) micro ATR-FTIR imaging using a microscope objective with Ge crystal and (ii) single reflection diamond ATR-FTIR imaging. Using both approaches the ATR-FTIR spectroscopic signatures allow the differentiation between several cellular organelles, e.g. nucleus and Endoplasmic Reticulum (ER). The overall cell shape can be defined by the distribution of the Amide II band in the measured image, while the DNArich nucleus and glycogen-rich ER could be imaged using spectral bands at $1084 \mathrm{~cm}^{-1}$ and $1023 \mathrm{~cm}^{-1}$, respectively. We also demonstrate the potential of ATR-FTIR spectroscopic imaging for unravelling the details of dynamic biological processes, which are not accessible from cell ensemble studies, with high molecular specificity and satisfactory spatial resolution. 


\section{INTRODUCTION}

The potential of vibrational spectroscopy for analysing biological objects in particular cells has been widely recognized. ${ }^{1,2}$ The great advantage of Infrared (IR) and Raman spectroscopy is their unprecedented molecular specificity and sensitivity to structural changes, which may often be correlated to the change in biological functions. Applications span from studies of protein folding via temperature jump with time resolved IR detection monitoring amide band vibrations ${ }^{3,4}$ to spectroscopic imaging of biological objects, including imaging at a single cell level, ${ }^{1,5-9}$ with both IR and Raman spectroscopy. FTIR spectroscopic imaging can be thought of as "chemical photography" due to its ability to take chemically specific snapshots of a sample. ${ }^{10}$ Spatially and timeresolved Raman spectroscopy was applied to study live yeast cells, ${ }^{11,}{ }^{12}$ fixed mammalian cells, ${ }^{9}$ as well as cell death (non-imaging study) ${ }^{13}$ in the natural aqueous environment. FTIR spectroscopic studies of aqueous samples have been hampered until recently by the strong absorbance of water bands in the regions of interest $(\mathrm{OH}$ stretching and bending bands at ca. $3600 \mathrm{~cm}^{-1}$ and $1650 \mathrm{~cm}^{-1}$ ). An additional restriction in the infrared region is imposed by intrinsically low spatial resolution of transmission measurements due to the diffraction limit for infrared light $(10-20 \mu \mathrm{m})$. Since IR spectroscopy can provide valuable information complementary to Raman spectroscopy there is a strong drive to move this technology forward. Recently the complications arising from water present in live biological samples have been successfully overcome by using the ultrabright synchrotron source for data acquisition. ${ }^{14,}{ }^{15}$ Subsequent subtraction of the water spectrum allowed the researchers to obtain IR spectral information on a single cell level 
in an aqueous environment. Although synchrotron infrared irradiation has been shown to have a minimal effect on living cells, ${ }^{15}$ the measurements are experimentally challenging, with additional complications arising from cell motility (migration out of the measurement beam). ${ }^{15}$

Apart from biological studies of fundamental interest, a significant research effort has been put into the development of FTIR or Raman spectroscopic methods in order to distinguish healthy tissue from malignant and pre-malignant samples for an early diagnosis of cancer. The motivation for this work comes from the fact that vibrational spectroscopic methods are fast, non destructive to tissue and rely on the intrinsic molecular vibrations of the molecules in the tissue and therefore do not require staining or addition of markers or labels. As such, vibrational spectroscopic methods are highly desirable for many clinical diagnostic applications.

The data available in the literature ${ }^{2,16,17}$ document the differences in vibrational fingerprints of the tissues collected from groups of healthy individuals and patients with clinically confirmed malignant modifications. Additionally, FTIR spectroscopy has been successfully used to detect the response of cultured cells to chemotherapeutic drugs, ${ }^{18,}, 19$ and single cell studies of apoptotic and necrotic cell death, including cell death induced by $\gamma$-irradiation, chemotherapeutics or toxic chemicals, ${ }^{20-22}$ aiming to establish the signatures of growing cancerous tissue versus successfully treated tissue. Thus, on the one hand, vibrational spectroscopic methods have potential in non destructive diagnosis of malignancy and, on the other hand, in detecting a patient's response to radiation- or chemotherapy in the clinic. 
Since synchrotron radiation is not readily accessible, most FTIR spectroscopic experiments on cell cultures, in particular those relevant to clinical studies, have been performed on dried samples to overcome strong water bands and afford the FTIR spectroscopic measurements in transmission mode. The dynamic processes, such as apoptosis or drug uptake, were studied by drying or fixing cultured cells at various treatment stages. However, this type of cell treatment poses significant disadvantages, since drying or fixing cells might lead to structural changes of key biological molecules. ${ }^{5}$ Therefore, this approach does not report on the state close to that found in live cells in an aqueous environment, which could lead to distortion of features characteristic of malignancy. In particular, drying and fixation of cultured cells is expected to affect the meaningfulness of the study of dynamic processes pertinent to the cell cycle and function of organelles. It is highly desirable to be able to study the live cell cultures with FTIR spectroscopy at a single cell level in a natural aqueous environment and furthermore to be able to detect the cell response to environmental stress in real time.

Attenuated Total Reflectance (ATR), the measurement of the attenuation to the IR beam caused by the absorption of the evanescent wave during the internal reflection, ${ }^{23}$ is the method of choice for studying samples in an aqueous environment. The effective pathlength of IR light in the sample is relatively small (a few micrometers) and is independent of the sample thickness. Therefore, minimal effort is required in the sample preparation. With this approach, absorbance of water bands will not saturate the signal received by the detector, thus allowing the analysis of other components that are within the depth of penetration of the evanescence wave (the depth of penetration is the distance at which the evanescent wave intensity exponentially decays to $1 / \mathrm{e}$ of its value at the 
interface), which is in the order of 1-2 $\mu \mathrm{m}$ with a diamond ATR crystal (refractive index: 2.4; angle of incidence: $45^{\circ}$ ) in the fingerprint region of the infrared spectrum. ${ }^{24}$ Our previous studies have shown that ATR-FTIR imaging is suitable to study the dissolution of pharmaceutical formulations in water, in situ, without the need of microtoming. ${ }^{10,25}$ Furthermore, using microscope objective with ATR crystal, for example Ge, it was possible to achieve a better spatial resolution than that available in transmission imaging approach (which is limited by diffraction of IR light in air). ${ }^{26,27}$ This enhanced resolution proved particularly useful in imaging of biomedical samples. We have also developed many applications using a ATR accessory with a diamond ATR crystal to obtain FTIR images of various samples, with relatively high spatial resolving power (resolving spatial features as small as $12 \mu \mathrm{m}),{ }^{24}$ that are otherwise difficult to obtain. ${ }^{25}$ Herein we report on the use of ATR FTIR imaging, as a method to study live cells in their natural aqueous environment with satisfactory spatial resolution without the need for synchrotron radiation.

\section{EXPERIMENTAL}

Two complementary approaches have been used providing flexibility with different fields of view and spatial resolution: (i) micro ATR-FTIR imaging using a microscope objective with Ge crystal and (ii) single reflection diamond ATR-FTIR imaging.

Micro ATR-FTIR imaging. Micro ATR images were measured using an infrared microscope equipped with a Ge micro ATR objective in conjunction to an infrared spectrometer (FTS-60A, Varian) and a 64 x 64 FPA detector. A spectral resolution of 8 
$\mathrm{cm}^{-1}$ and 128 scans was used. The micro ATR element can be removed from the objective and used for direct cultivation of cells on the measuring surface. A mini chamber as described in Fig. 1 has been created to hold a small amount of liquid (ca. $0.5 \mathrm{ml}$ ) at the measuring surface of the micro ATR crystal. All equipment used was sterilised with $99 \%$ ethanol. The Ge crystal was coated with $0.01 \%$ poly-L-lysine (Sigma-Aldrich, UK) to improve cell adhesion. The background spectra were collected with clean Ge crystal.

Diamond ATR-FTIR imaging. A diamond ATR accessory was carefully aligned in a large sample compartment. The sample compartment was attached to the same spectrometer and FPA detector used for the micro ATR imaging experiments. The spectrometer was also operating in continuous scan mode with spectra measured at $4 \mathrm{~cm}^{-1}$ resolution and 256 scans. The top plate of the diamond ATR accessory can be detached and placed in the incubator for the direct cultivation of cells on the measuring surface. A small chamber of diameter ca. $15 \mathrm{~mm}$ was fixed on the top plate. The chamber holds ca. 5 $\mathrm{ml}$ of solution with the diamond in the centre of the chamber bottom (Fig. 1). The diamond surface was coated with $0.01 \%$ poly-L-lysine to improve cell adhesion. The background spectra were collected with clean diamond crystal. Spectral and spatial masking was performed using the Varian Resolution Pro (Varian) and ISys software (Malvern).

Cell cultures. Epithelial cell line SK-OV-3 (human ovarian carcinoma, ECACC) was grown at $37^{\circ} \mathrm{C}$ under the $5 \% \mathrm{CO}_{2}$ atmosphere in Dulbecco's modified Eagle's medium (DMEM) supplemented with $10 \%$ foetal bovin serum and $5 \%$ penicillin/streptomycin antibiotics (Invitrogen, UK). Cells were passaged when 70-90\% confluent. The suspension of cells was seeded on the measuring surface of the ATR crystal at a 
concentration of $1 \times 10^{5}$ cells $/ \mathrm{ml}$. Only well adhered cells were detected in ATR experiments due to small penetration depth (a few $\mu \mathrm{m})$. The cell death was induced by either single injection of buffered solution of potassium cyanide (giving $1 \mathrm{mM}$ as the final concentration of $\mathrm{KCN}$ ) or exchange of the culture medium with equivalent volume of PBS. The viability of attached cells was confirmed by the lack of the membrane permeability to tryphan blue (Sigma-Aldrich, UK).

Fluorescence imaging was performed using a confocal laser scanning microscope (Leica TCS SP2), coupled to a CW argon-ion laser (488 nm). The fluorescence emission of the ER tracker green (BODIPY® FL $\mathrm{C}_{5}$-ceramide complexed to BSA, Molecular Probes) in the cells was spectrally dispersed using a prism and detected using a photomultiplier tube through water immersion $63 \times$ objective $(\mathrm{NA}=1.2)$.

\section{RESULTS AND DISCUSSION}

The schematic of the ATR-FTIR imaging experimental setup, developed in this work, is shown in Fig. 1. Human cancer cells are seeded and grown directly on the ATR crystal, coated with a thin layer (without noticeable spectral contribution) of poly-L-lysine to allow effective cell adhesion. A significant variation in the quality of contact between the cell and the crystal would be reflected in the difference of the absorbance of the water bands, which is not the case for the area where the cell was attached to the crystal. We believe that this is because the contact between the cell and the crystal was good. Apparently, adhesion of the live cell proceeds via the interaction between the membrane and a very thin layer of poly-L-lysine._The aqueous layer of the cell culture medium 
maintains the natural environment around adherent cells and can be also used as a medium to administer chemicals. While cells are maintained in aqueous environment, the abundance of water does not affect the IR spectra obtained from the adherent cells since the penetration depth of IR light does not go beyond the thickness of the cell while it goes deep enough to interrogate chemicals inside the cell through the cell membrane (ranging between $\sim 1$ to $2 \mu \mathrm{m}$ at $1050 \mathrm{~cm}^{-1}$ depending on the ATR method used, which is significantly greater compared to the several nanometres thick cell membrane). Nevertheless, the intrinsic limitation of the ATR approach in the infrared region is that it will not probe components of the cell that may reside beyond few micrometers from the surface of the crystal; this may be particularly important if the cells were not well adhered to the surface of ATR crystal.

We first aimed to establish whether the ATR imaging method allows to differentiate between different cellular organelles. For this purpose we have used the micro ATR with Ge crystal (refractive index: 4), which provides the highest spatial resolution $(2-4 \mu \mathrm{m})$ using FTIR imaging ${ }^{26,27}$ and the penetration depth of ca. $1 \mu \mathrm{m}$ at $1050 \mathrm{~cm}^{-1}$ with the angle of incidence of ca. $30^{\circ}$. We have analysed the IR spectra of cells in the 1700-1000 $\mathrm{cm}^{-1}$ spectral region (Fig. 2, top) and several bands were identified, which could potentially be used to differentiate between cellular organelles.

Amide I band $\left(\sim 1650 \mathrm{~cm}^{-1}\right)$ is characteristic of proteins, however this band position heavily overlaps with the intense absorbance band of water (bending mode) in this region as water is a major component within the live cell. In the present work Amide II band, 1506-1574 $\mathrm{cm}^{-1}$, was used instead of Amide I band to image the location of proteins due to the minimal contribution from water in this region. The image in Fig. 2, left was 
generated by plotting the distribution of the integrated absorbance of the Amide II band at each pixel across the imaged area. Since relatively high concentration of proteins is found throughout the cell, we expect that the image based on Amide II band will show the location of adhered cells on the imaging surface i.e. the cell contour (the area of attached cell),. The resulting image indicates that adherent cells cover most of the imaged area, consistent with visual inspection (in transmission mode) through the microscope objective. Based on the analysis of the shape of the protein distribution, there are 3 cells captured within the imaging area on Fig. 2 and the size of each cell is approximately 30$50 \mu \mathrm{m}$, consistent with the size expected of a SK-OV-3 cell.

We have also created an image based on the integrated area of the band at $1084 \mathrm{~cm}^{-1}$, due to the DNA/RNA phosphate backbone stretching vibration. ${ }^{28}$ The resulting image is shown in Fig. 2, middle. While there is a significant overlap between the Amide II- and $1084 \mathrm{~cm}^{-1}$-based images, it is clear that the absorbance of $1084 \mathrm{~cm}^{-1}$ band is not homogeneously distributed through the area defined by the cell contour (the area of attached cell) and shows an increased absorbance in certain cell areas. This can be explained by the high concentration of DNA in the cell nucleus. From these data we conclude that the location of the nucleus can be identified as the area where the absorbance of the $1084 \mathrm{~cm}^{-1}$ band is the strongest (depicted by two white arrows in the image). Phospholipids can also contribute to the absorbance in this region and this is why we observe non-zero absorbance at $1084 \mathrm{~cm}^{-1}$ outside nucleus in the cell (where the highest concentration of phosphates is present, characterised by the highest absorbance of the band at $1084 \mathrm{~cm}^{-1}$ ). 
Additionally we have analysed the absorbance variations of $1023 \mathrm{~cm}^{-1}$ band through the image, which is not due to proteins or DNA. We have established that the relative absorbance of $1023 / 1084 \mathrm{~cm}^{-1}$ and $1023 / 1550 \mathrm{~cm}^{-1}$ bands vary significantly throughout the cell image. Previous non-imaging study has employed the $1030: 1080 \mathrm{~cm}^{-1}$ ratio as a marker for the metabolic turnover of cells. ${ }^{29}$ We have also established that the strongest absorbance of the $1023 \mathrm{~cm}^{-1}$ band is found in the perinuclear area (see Fig. 2, right) and the spectrum extracted from this area corresponds closely to the IR spectrum of glycogen, ${ }^{30}$ Fig. 3.

Glycogen is the polysaccharide which is known to play an important role in the glucose cell cycle and the energy storage for cells. Glycogen is known to be present in high levels in several cell types, in particular liver cells $(8 \%)$, but also in epithelial cells of cervix and breast. ${ }^{31,} 32$ The abnormal level of glycogen in tissues has been previously detected and linked to an abnormality or disease, e.g. starvation in animals, ${ }^{31}$ diabetes in human patients, or indication of neoplastic disease, detected by FTIR spectroscopy. ${ }^{2}$ It is therefore of clear benefit to be able to image the glycogen content on the single cell level in live cells without the need for using the staining assay. More importantly, this opens up the possibility to study the dynamics of the cellular processes involving glycogen metabolism, unavailable with any other existing methods. Glycogen is known to accumulate in a cell in granules together with the enzymes necessary for its synthesis and degradation. There are several reports linking the site of storage of glycogen granules in the cell with endoplasmatic reticulum (ER). ${ }^{31}$ Thus we tentatively assign the area with the strongest absorbance of the $1023 \mathrm{~cm}^{-1}$ band to ER, Fig. 2, right. This is the first example of imaging of ER in a live cell using vibrational spectroscopy-based imaging approach. 
To ascertain that the ATR-FTIR-based images of SK-OV-3 cells can indeed distinguish between different cellular organelles, we have recorded confocal fluorescence images of SK-OV-3 cells stained with ER tracker, Fig. 4. While this marker stains ER (shown in orange) and to a certain extent cellular cytosol (shown in green), it does not stain the nucleus (which appears blue, the same colour as surrounding medium). There are clear similarities between the fluorescence-based images of SK-OV-3 cells and the ATR-FTIR images of cells created based on the analysis of selected IR bands. The image generated using the $1084 \mathrm{~cm}^{-1}$ band in Fig. 2 share similarity with the image of the nucleus which is typically characterised by the oval shape with a cross section of ca 15-20 $\mu \mathrm{m}$. Since the $1084 \mathrm{~cm}^{-1}$ band has been previously assigned as the $\mathrm{PO}_{2}{ }^{-}$symmetric stretch from DNA, ${ }^{28}$ our conclusions appears sound as nucleus contains the highest concentration of DNA in a cell.

The brightest area based on the absorbance of $1023 \mathrm{~cm}^{-1}$ band lies closely to the area occupied by the nucleus in all the cells studied (see Fig. 3 and Fig. 5) and strongly resembles the shape of ER, when compared to the fluorescence image in Fig. 4. As expected, Amide II band appears to image the cytosol of the cell, consistent with reports existing in the literature that the absorbance of Amide I and II bands $\left(1660\right.$ and $1550 \mathrm{~cm}^{-}$ ${ }^{1}$ ) does not significantly vary for different cellular organelles. ${ }^{33}$ Note that the irregular shape of this larger area is consistent with the shape observed for viable SK-OV-3 cells in fluorescence imaging. Thus the comparison between the confocal fluorescence image of cells and the micro ATR-FTIR spectroscopic images obtained using the bands at 1550, 1084 and $1023 \mathrm{~cm}^{-1}$ validates the assignment of these bands to the different types of structures in the cell. 
Once we have established the ability of ATR imaging to distinguish between different organelles in live cells, it becomes possible to study the in situ response of live cells to a change in their environment, e.g addition of toxic chemicals or other forms of stress. Since glycogen is thought to be responsible for the energy storage cycle in a cell, we have chosen to study the effect of depriving the cell of the vital medium components, by exchanging the normal cell medium (DMEM) to a buffered phosphate saline solution, PBS. We have then monitored the response of cells within the next three hours, Fig. 5. In this experiment it was useful to test a different ATR approach with a larger field of view and somewhat lower spectral resolution (diamond ATR). In this approach, ${ }^{25}$ diamond is used as the ATR element which images an area of ca. $0.5 \mathrm{~mm} \times 0.6 \mathrm{~mm}$ with a spatial resolution of $15-20 \mu \mathrm{m}$ allowing many individual cells to be imaged simultaneously. Statistically this should allow us to observe the changes in cells with different initial contents of glycogen and/or a different rate of changes associated with removal of nutrients.

The series of images taken following an exchange of culture medium to PBS, simultaneously showing the transformation of the areas taken by protein $\left(1^{\text {st }}\right.$ column of image from the left), nucleus $\left(2^{\text {nd }}\right.$ column $)$, ER ( $3^{\text {rd }}$ column), and the ratio of the $3^{\text {rd }}$ column image over the $2^{\text {nd }}$ column image $\left(4^{\text {th }}\right.$ column $)$ over time are shown in Fig. 5. There are no major changes to the image of the protein or DNA distributions. However, the more drastic and faster changes occur to the area characterized by the high presence of glycogen, assigned to ER. It is clear that there is a marked decrease in glycogen concentration after the exchange of the cell culture medium with PBS. This rapid change 
can be better monitored by examining the image obtained from the ratio of the 1023/1084 bands (Fig. $5,4^{\text {th }}$ column). These data clearly indicate that the content of glycogen in a cell decreases rapidly compared to the DNA content. The disappearance of glycogen-rich domains continues until the cells start to detach from the measuring surface ( 3 hours after the medium exchange). The ratiometric image $\left(4^{\text {th }}\right.$ column $)$ also demonstrates that the reduction in the absorbance of the band at $1023 \mathrm{~cm}^{-1}$ is not due to cell detachment, since the absorbance of band at $1084 \mathrm{~cm}^{-1}$ remains virtually unchanged. In addition we confirmed that the cells under study are still viable during the course of starvation by trypan blue exclusion assay.

We have also studied the effects of external stress on live cells such as KCN poisoning, causing the malfunction of mitochondria and leading to cell death. The series of images in Fig. 6 have shown that the absorbance of all corresponding spectral bands increased immediately after the addition of KCN solution to the vessel. The cause of the increase in absorption is still unknown. Nevertheless, it is clear that over the course of continuous observation, between 10 and 50 minutes following $\mathrm{KCN}$ addition, all bands show the small reduction in absorbance, which we assign to slow partial cell detachment. In a separate set of experiments (data not shown) where $\mathrm{KCN}$ was not added, the cells remain adherent to the measurement surface of the ATR crystal over a much longer period (several hours). We have detected no major changes in the spectrum of human cancer cells during the $\mathrm{KCN}$ poisoning, which is consistent with the results of recent conventional ATR-FTIR spectroscopic study (ZnSe crystal) of cell death. ${ }^{34}$ These results are complementary to the literature data based on $\mathrm{KCN}$ poisoning of bacterial cell monitored with Raman spectroscopy. ${ }^{11,12}$ 
The available literature reports indicate that only small differences are observed between the IR spectra of different cellular organelles, mostly reflecting the variation in sample thickness, ${ }^{33}$ even though the spectra of proteins, DNA, RNA and lipids are distinguishable. ${ }^{35}$ If correct, this observation diminishes the meaningfulness of IR imaging of single cells and makes observations of the mechanism of intracellular processes with IR spectroscopy virtually impossible. However, we have noted that these conclusions have been mainly reached in the FTIR spectroscopic studies of dried samples, which are deprived of signatures of life and are not capable of providing dynamic information pertinent to a live cell cycle. We have demonstrated that it is possible to capture FTIR images of live cells in their natural environment with relatively high resolution. We have also demonstrated the possibility to chemically image live cancer cells in situ under chemical or other treatment which opens up a range of opportunities for studying the effect of different drugs on live adherent cells.

\section{CONCLUSIONS}

The ATR-FTIR spectroscopic imaging approach was applied to study human live cancer cells. This approach is limited to the probing depth of a few micrometers and can not probe components of the cell that may reside deeper from the surface of the crystal. However, it is important that this limitation is not significant in the case of cells that are well-adhered to the crystal coated with a very thin layer of poly-L-lysine. This allowed us to study live human cancer cells in an aqueous environment, on a single cell level. We have successfully applied this advantage to monitor the decrease in the intracellular concentration of glycogen during cell starvation; the remarkable observation on the single 
cell level, which resembles what have been observed previously in liver tissue in animal models. ${ }^{31}$ Additionally, this methodology has potential implications for learning more about cancer pathology as low levels of glycogen have been detected in dried breast tissue with confirmed malignancy. The small differences in IR spectra at various stages of cell cycle or under conditions of lack of nutrients or cell confluency have been noted

previously with IR microspectroscopy ${ }^{35,36}$ however these are shown with exceptional clarity using the presently reported approach at a single cell level. Thus, the methodology outlined in this report is accessible and could provide a convenient method for optimisation of chemotherapy approaches and drug testing in the clinic.

\section{ACKLOWLEGMENTS}

We thank EPSRC for support of this work and Life Science Interface Fellowships awards to MKK (EP/E038980/1) and to KLAC (EP/D066859/1). The fluorescence images were obtained in Dr Klaus Suhling's laboratory (King's College London) and his assistance is gratefully acknowledged. 


\section{REFERENCES}

1. M. Diem, M. Romeo, S. Boydston-White, M. Miljkovic, and C. Matthaus, Analyst 129, 10, 880 (2004).

2. M. A. Cohenford and B. Rigas, Proc. Natl. Acad. Sci. U. S. A. 95, 26, 15327 (1998).

3. S. Williams, T. P. Causgrove, R. Gilmanshin, K. S. Fang, R. H. Callender, W. H. Woodruff, and R. B. Dyer, Biochemistry 35, 3, 691 (1996).

4. R. B. Dyer, F. Gai, and W. H. Woodruff, Accounts Chem. Res. 31, 11, 709 (1998).

5. M. Romeo, C. Matthaus, M. Miljkovic, and M. Diem, Biopolymers 74, 1-2, 168 (2004).

6. M. Miljkovic, M. Romeo, C. Matthaus, and M. Diem, Biopolymers 74, 1-2, 172 (2004).

7. P. Lasch, A. Pacifico, and M. Diem, Biopolymers 67, 4-5, 335 (2002).

8. C. Matthaus, S. Boydston-White, M. Miljkovic, M. Romeo, and M. Diem, Appl. Spectrosc. 60, 1, 1 (2006).

9. $\quad$ N. Uzunbajakava and C. Otto, Opt. Lett. 28, 21, 2073 (2003).

10. S. G. Kazarian and K. L. A. Chan, Macromolecules 36, 9866 (2003).

11. Y. S. Huang, T. Karashima, M. Yamamoto, and H. Hamaguchi, Biochemistry 44, 30, 10009 (2005).

12. Y. S. Huang, T. Karashima, M. Yamamoto, T. Ogura, and H. Hamaguchi, J. Raman Spectrosc. 35, 7, 525 (2004).

13. S. Verrier, I. Notingher, J. M. Polak, and L. L. Hench, Biopolymers 74, 1-2, 157 (2004).

14. N. Jamin, P. Dumas, J. Moncuit, W. H. Fridman, J. L. Teillaud, G. L. Carr, and G. P. Williams, Proc. Natl. Acad. Sci. U. S. A. 95, 9, 4837 (1998).

15. D. A. Moss, M. Keese, and R. Pepperkok, Vib. Spectrosc. 38, 1-2, 185 (2005).

16. C. P. Schultz, K. Z. Liu, J. B. Johnston, and H. H. Mantsch, J. Mol. Struct. 408, 253 (1997).

17. B. R. Wood, M. A. Quinn, B. Tait, M. Ashdown, T. Hislop, M. Romeo, and D. McNaughton, Biospectroscopy 4, 2, 75 (1998).

18. J. Sule-Suso, D. Skingsley, G. D. Sockalingum, A. Kohler, G. Kegelaer, M. Manfait, and A. El Haj, Vib. Spectrosc. 38, 1-2, 179 (2005).

19. H. Y. N. Holman, R. Goth-Goldstein, M. C. Martin, M. L. Russell, and W. R. McKinney, Environ. Sci. Technol. 34, 12, 2513 (2000).

20. N. Gault, J. L. Poncy, and J. L. Lefaix, Can. J. Physiol. Pharmacol. 82, 1, 38 (2004).

21. K. Z. Liu, L. Jia, S. M. Kelsey, A. C. Newland, and H. H. Mantsch, Apoptosis 6, 4, 269 (2001).

22. N. Jamin, L. Miller, J. Moncuit, W. H. Fridman, P. Dumas, and J. L. Teillaud, Biopolymers 72, 5, 366 (2003).

23. N. J. Harrick, Internal reflection spectroscopy (Harrick Scientific Corporation, New York, 1987), Third ed.

24. K. L. A. Chan, F. H. Tay, G. Poulter, and S. G. Kazarian, Appl. Spectrosc. 62, 10, 1102 (2008). 
25. S. G. Kazarian and K. L. A. Chan, Biochim. Biophys. Acta.-Biomem. 1758, 7, 858 (2006).

26. K. L. A. Chan and S. G. Kazarian, Appl. Spectrosc. 57, 4, 381 (2003).

27. C. S. Colley, S. G. Kazarian, P. D. Weinberg, and M. J. Lever, Biopolymers 74, 4, 328 (2004).

28. Y. Mao, L. N. Daniel, N. Whittaker, and U. Saffiotti, Environ. Health Perspect. 102, 165 (1994).

29. E. Gazi, J. Dwyer, P. Gardner, A. Ghanbari-Siahkali, A. P. Wade, J. Miyan, N. P. Lockyer, J. C. Vickerman, N. W. Clarke, J. H. Shanks, L. J. Scott, C. A. Hart, and M. Brown, J. Pathol. 201, 1, 99 (2003).

30. L. Chiriboga, P. Xie, H. Yee, V. Vigorita, D. Zarou, D. Zakim, and M. Diem, Biospectroscopy 4, 1, 47 (1998).

31. J. C. H. de Man and A. P. R. Blok, J. Histochem. Cytochem. 14, 135 (1966).

32. R. Geddes, Biosci. Rep. 6, 5, 415 (1986).

33. P. Lasch, M. Boese, A. Pacifico, and M. Diem, Vib. Spectrosc. 28, 1, 147 (2002).

34. W. Yang, X. Xiao, J. Tan, and Q. Cai, Vib. Spectrosc. doi:10.1016/j.vibspec.2008.1004.1016 (2007).

35. J. R. Mourant, Y. R. Yamada, S. Carpenter, L. R. Dominique, and J. P. Freyer, Biophys. J. 85, 3, 1938 (2003).

36. A. Pacifico, L. A. Chiriboga, P. Lasch, and M. Diem, Vib. Spectrosc. 32, 1, 107 (2003). 


\section{Figure Legends:}

Fig. 1: Schematic diagram of diamond ATR FTIR imaging setup for the live cell imaging measurements.

Fig. 2: Top: A typical spectrum of cells recorded in the protein-rich region; insert shows expanded "fingerprint" region of the spectrum. Bottom: micro ATR FTIR images of cells. The left, middle and right images were generated based on the Amide II band, $1084 \mathrm{~cm}^{-1}$ band and $1023 \mathrm{~cm}^{-1}$ band respectively. White area indicates a high absorbance while dark area indicates otherwise. Each image size is $64 \times 64 \mu \mathrm{m}^{2}$.

Fig. 3:Averaged spectra extracted from the glycogen-rich region (black solid line, assigned to ER) and the DNA-rich region of the cell (dotted line, assigned to the nucleus).

Fig. 4: Fluoresence image of SK-OV-3 cells incubated with ER tracker® Green (Molecular Probes), obtained following $488 \mathrm{~nm}$ excitation, 525 $\pm 20 \mathrm{~nm}$ emission detection. False colour scale applied. ER in each cell appears bright orange, while the cell cytosol appears green. The nucleus is not stained by this marker and appears blue. The size of the image is $114 \times 114 \mu \mathrm{m}^{2}$.

Fig. 5: ATR FTIR images of SK-OV-3 cells starvation, achieved by exchanging DMEM medium to PBS, measured on a diamond ATR imaging accessory. From the left, 
image column 1 to 4 were generated based on the Amide II, $1084 \mathrm{~cm}^{-1}$ band, 1023 $\mathrm{cm}^{-1}$ band and the ratio of $1023 / 1084$ bands. Image size is ca. $0.5 \mathrm{~mm} \times 0.6 \mathrm{~mm}$.

Fig. 6: ATR FTIR images of live SK-OV-3 cell before (time $=0 \mathrm{~min}$ ) and after exposure to DMEM containing $1 \mathrm{mM} \mathrm{KCN}$, measured on a diamond ATR imaging accessory. Image size is ca. $0.5 \mathrm{~mm} \times 0.6 \mathrm{~mm}$. 
Chamber

Cell seeded in media

(0)

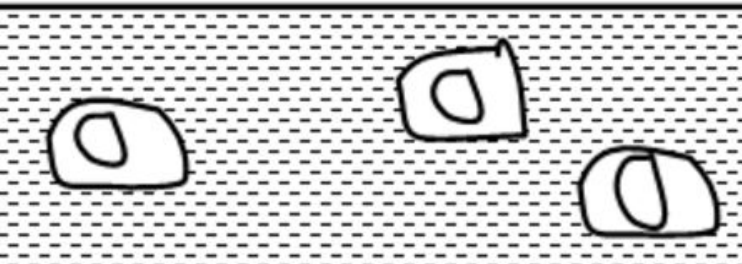

(0)

(o)

(o)

(0)

(a)

(a)

(0) (ै)

(o)

(o)

(a)

Diamond top plate

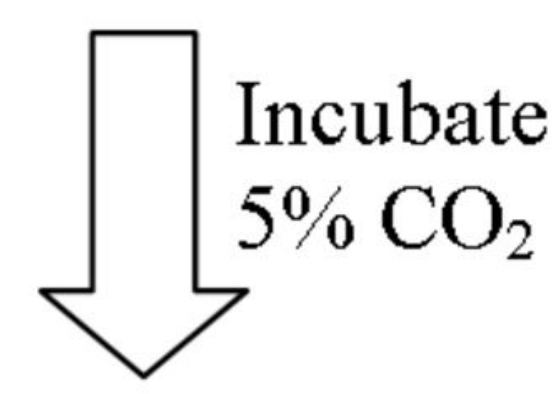

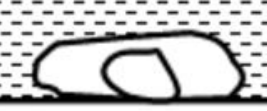

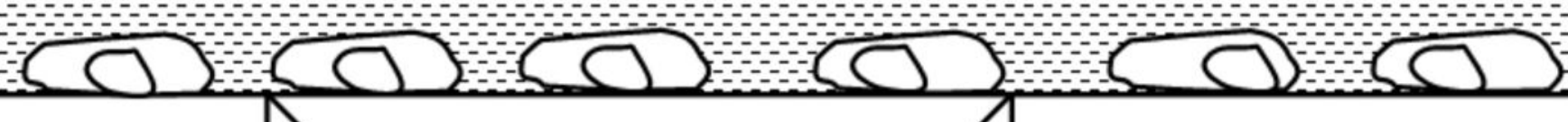

IR beam

ATR
crystal

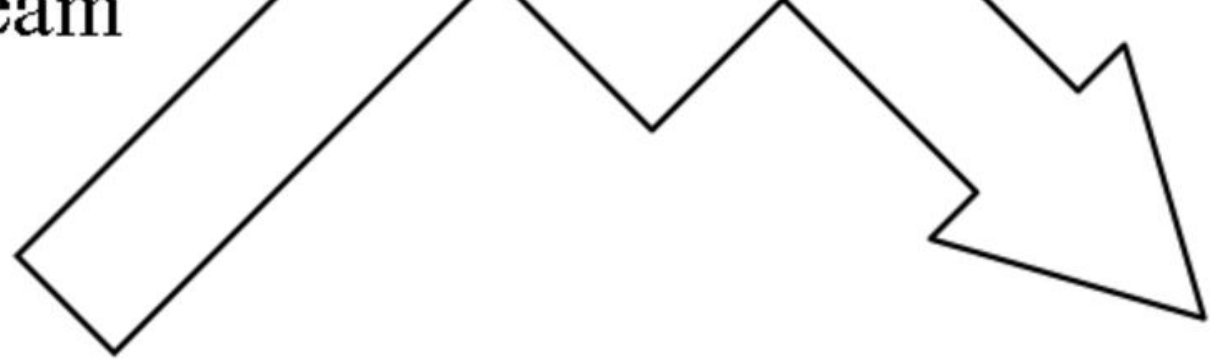

FPA

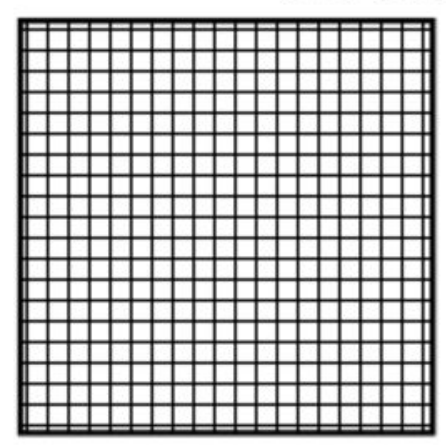









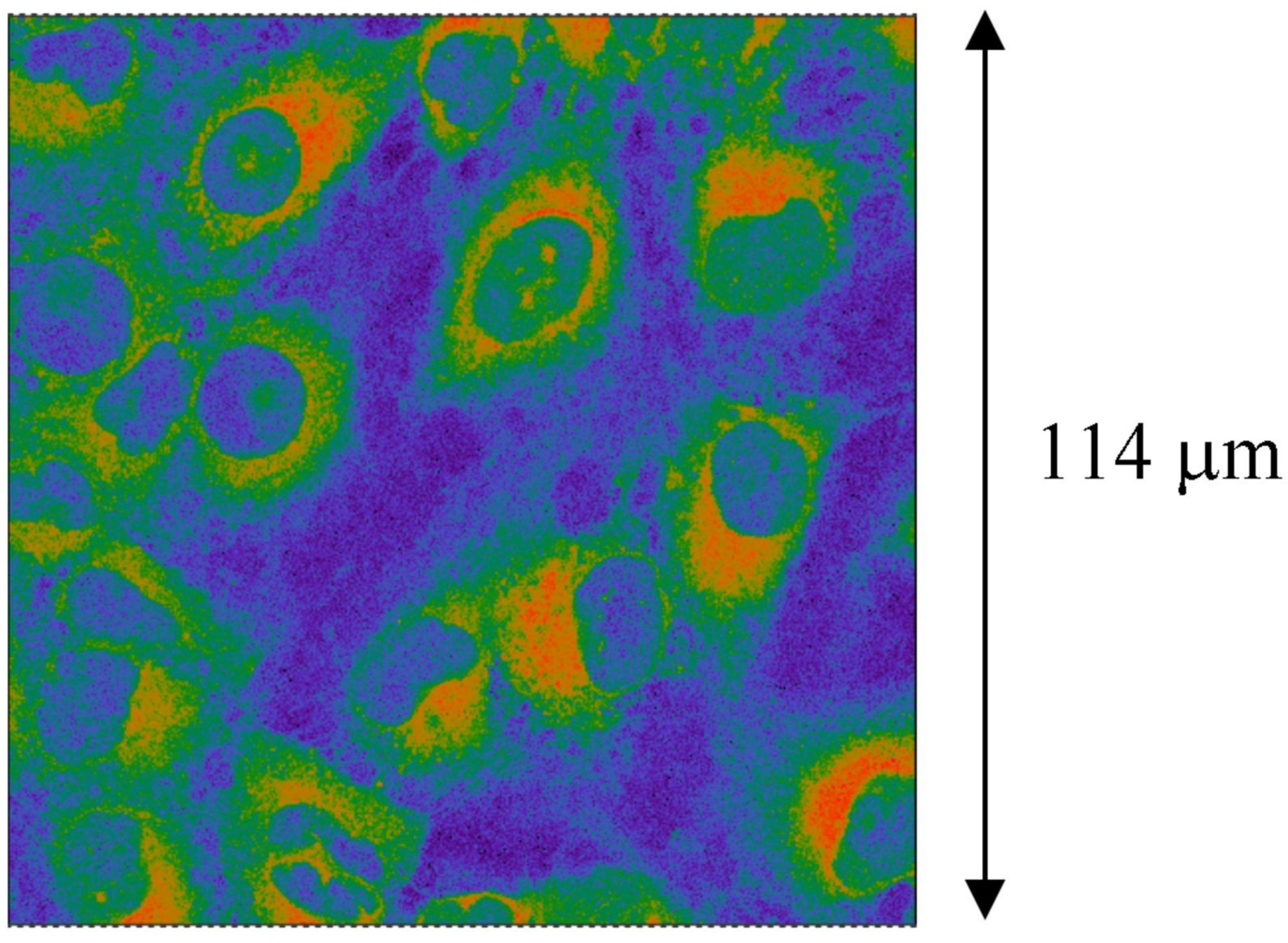


